\title{
Brownian Gyrator: An Experimental Realization
}

\author{
Jalpa Sonia ${ }^{\mathrm{a},}$, Aykut Argun ${ }^{\mathrm{b}}$, Lennart Dabelow ${ }^{\mathrm{c}}$, Stefano Bo ${ }^{\mathrm{d}}$, Ralf Eichhorn ${ }^{\mathrm{d}}$, Giuseppe Pesce ${ }^{\mathrm{e}}$ and Giovanni \\ Volpe $\mathbf{a}^{\mathbf{a}, \mathbf{b}}$ \\ ${ }^{a}$ Soft Matter Lab, Department of Physics, Bilkent University, Ankara, Turkey, \\ ${ }^{b}$ Department of Physics, University of Gothenburg, Gothenburg, Sweden, \\ 'Nordita, Royal Institute of Technology and Stockholm University-Roslagstullsbacken, Stockholm, Sweden \\ ${ }^{d}$ Friedrich Schiller University, Jena, Germany \\ e Dipartimento di Fisica, Universita degli Studi di Napoli, Complesso Universitario Monte S. Angelo, Napoli, Italy \\ *Corresponding author: jalpaben.soni@bilkent.edu.tr
}

\begin{abstract}
We present an experimental realization of a minimal heat engine in the form of a single Brownian particle, performing gyrating motion by systematic torque generation due to dissipation from two different heat baths in a simple optical tweezer set-up. OCIS codes: (000.6850) Thermodynamics; (020.7010) Laser trapping
\end{abstract}

Heat engines have been one of the most intriguing research topics in modern thermodynamics owing to their versatility and wide range of applications. Recently, there has been a new-found boost of interest in them following from the advancement of nanotechnology. With the ever-expanding perspectives of micro- and nano-devices, it is interesting to look for ways to realize heat engines at these scales. One such system was theoretically conceptualized recently in the form of a Brownian gyrator [1]. When a Brownian particle is confined in a generic optical potential with two different heat baths acting on it, the asymmetry of the thermal and restoring forces leads to the generation of torques, making it act as a microscopic heat engine. We have shown that indeed it is possible to experimentally realize such a simple microscopic heat engine. We optically trap a Brownian particle in an elliptic potential [2], and apply different effective temperatures in two orthogonal directions of the trap. We imitate the thermal noise by applying a randomly fluctuating electric field along one direction [3], thus creating two different heat baths. Under this condition, the Brownian particle exhibits rotational motion, the characteristic properties of which depend upon the strength of the temperature asymmetry along the two directions as well as the angle between potential asymmetry axes and temperature asymmetry axes. We quantify the rotational motion by calculating the generated torque from the experimentally measured particle-trajectories. We show good agreement between theoretical prediction and experimental results.
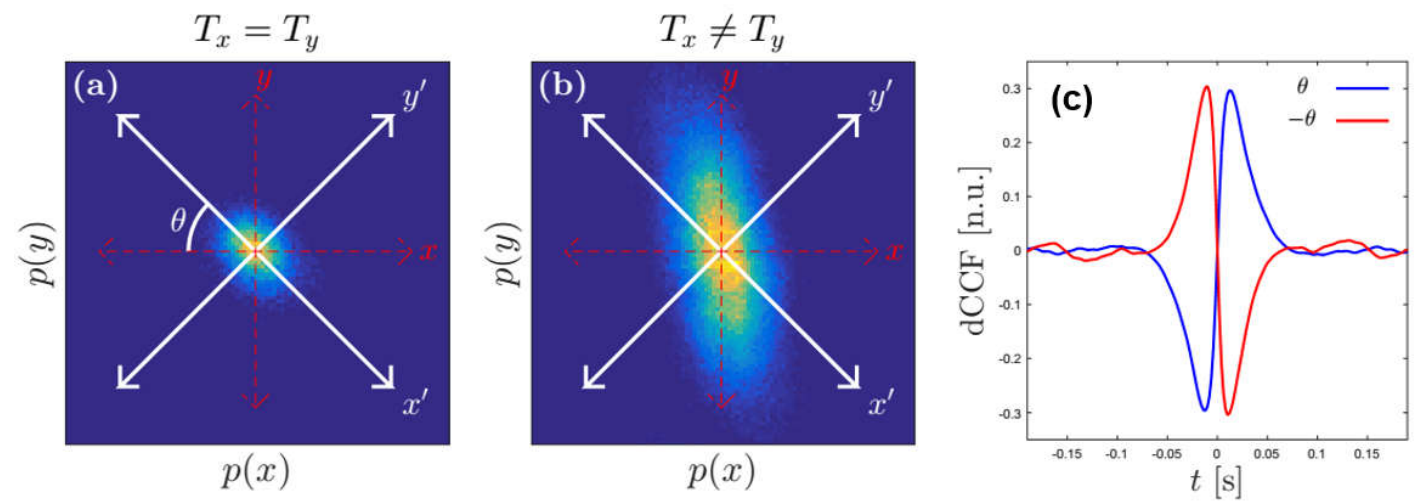

Figure 1: (a) Probability distribution of a Brownian particle inside an elliptical potential. Fundamental axes of this elliptical potential is $\mathrm{x}^{\prime}$ and $\mathrm{y}^{\prime}$, which is rotated from original $\mathrm{x}$ and $\mathrm{y}$ by $\theta$. (b) The distribution of the same particle under the same trap when the effective temperature along y axis is increased, and (c) Differential cross correlation function shown for a particle trapped in an elliptical trap oriented at $\theta=45^{\circ}$ (blue lines) and $\theta=45^{\circ}$ (red lines), in the presence of electric field showing the rotation of the Brownian gyrator.

[1] R. Filliger and P. Reimann, "Brownian Gyrator: A Minimal Heat Engine on the Nanoscale”, Phys. Rev. Lett. 99, 230602 (2007).

[2] P. Jones, O. Maragó, and G. Volpe. Optical tweezers: Principles and applications. Cambridge University Press, (2015).

[3] G Volpe, G Volpe and D Petrov: "Brownian motion in a nonhomogeneous force field and photonic force microscope", Phys. Rev. E 76, 061118 (2007). 